\title{
Flight manoeuvring and safe flight visualization with the aid of wide-ranging scrutiny and automation software
}

\author{
J V Muruga Lal Jeyan ${ }^{1}$, Jency Lal, ${ }^{2,}$, M Senthil Kumar $^{3}$, and Arfaj Ahamed Anwar ${ }^{4}$ \\ ${ }^{1}$ Department of Aerospace, Lovely Professional University, India \\ ${ }^{2}$ Research Scholar, Department of Aerospace, Prist University, India \\ ${ }^{3}$ Research Supervisor, Department of Aerospace, Prist University, India \\ ${ }^{4} \mathrm{MRB}$ Engineer - United technologies Aerospace system - Ontario, L6L5Y7Canada
}

\begin{abstract}
This document guides to an efficient use of FDVCAS system. FDVCAS is a reconstruction, playback, interactive visualization tool, which collects, stores, processes, analyses and present the flight data in high fidelity graphical presentation in $2 \mathrm{D}$ and $3 \mathrm{D}$ format. It synchronizes and presents in both graphical and synthetic visual form to the extends of analysing the impact of the data on the system in 3D graphic animation. The existing process involves in capturing the data from different input sources and formats, analysing the features of the data monitoring is done manually in offline in a unsynchronized fashion, This is difficult, time consuming and requires highly skilled technical expertise as it is visualized in plain data form. The above standard procedures followed shall be integrated and automated with minimum intelligence to be built, in with the system. In order to analyse the flight data in a coherent way it is required to integrate both $2 \mathrm{D}$ and $3 \mathrm{D}$ form The proposed concept is the Development of a system with a single solution by providing data display, graphical charts and replay features with a sophisticated graphical user interface, which is super imposed on the outside window imagery. FDVCAS consists of Bypass data acquisition system, Outside Window Imagery (OWI) system with graphical analyser. Bypass data acquisition system receives data from aircraft recorder and transmits the data in engineering format to FDVCAS system. The OWI system has three main modules namely, 3D Visual module, GUI based Graphical analyser and Warning display module. The warnings and the graphical plots are super imposed on the 3D Visual.
\end{abstract}

\section{Review \& observation}

The data from an aircraft is acquired using flight data recorder and analysed to identify any exceedances of the data and failures of the sub systems. The data analyzation presently used is namely "data display" to compare the selective data and its range. The capability of comparison and analysis of data are limited to the options provided for analysis in the above tools. The 3D visualization of the flight path is done separately through "synthetic

*Corresponding author: jencymlal@gmail.com 
terrain visualization software" developed by ADA. During the visualization, the abnormal behaviours in the phases of the flight are identified with the list of possible parameters and the respective data are analysed using above said methods [1-4]. The above standard procedures followed shall be integrated and automated with minimum intelligence to built, in with the system. In order to analyse the flight data in a coherent way it is required to integrate both 2D and 3D form in a synchronized way. Analysing and comparison of the problem in a data form was very difficult since the decision is made based on behaviour of data [5-7]. The requirements were analysed using standard tools called MS ACCESS and MSWord to read and to display the data in offline format, the 3D visualization is done using Synthetic Terrain Visualization Tool [8-10]. The process involves capturing the data from different input sources and extracting the data from different data formats, analysing the features of the data and monitoring are done manually in an unsynchronized fashion which is difficult and requires highly skilled technical expertise as it is visualized in plain data form [11-14]. In order to analyse the data effectively it is required to develop a system with graphical user interface to monitor the data in offline. To overcome the above difficulties a proper analysing software is required so FDVCAS system is used.

\section{Problem definition}

Aerospace plays a significant role in analysing flight data for each flight sortie. During take-off and landing, sensors mounted on the aircraft continuously measure critical parameters such as acceleration, external pressure, internal engine performance, fuel tank pressure, fuel temperature, guidance and navigation performance etc [15] are stored in a recorder. These parameters vary with phases of the flight time - some of them quite rapidly. Acceleration, for example, can oscillate up to several thousand times each second. Flight data, extracted directly from the aircraft's Flight Data Recorder (FDR) is routinely analysed to identify the areas of risk and opportunities for improved efficiency $[5,8]$. As the number of sortie increases the data becomes huge and difficult to analyse and takes more time. Analysing the problem in a data form is also very difficult since the decision is made based on behaviour of data. In order to analyse the data effectively it is required to develop a system with graphical user interface to monitor the data in online, which is super imposed on the synthetic outside window imagery. The analyzation of the data pictorially improves the efficiency and helps to study the impact of the data on the system. The extracted data from the recorder is stored in a data file in the form of comma separator variable format and the same is given as input to FDVCAS. The list of variables to be monitored are selected through GUI and displayed in the user selected graphics format.

\subsection{Hardware \& software specification}

The "synthetic terrain visualization" module developed by ADA consists of south Asian terrain data with five-meter resolution with the exact height information. The main process involved during display of every frame is culling the area depends on the present aircraft position and its orientation and rendering the image on the screen, which requires high computing power and graphics capability. Every frame of the image also required to be updated at minimum $60 \mathrm{~Hz}$. The hardware configuration selected to realize the same based on the above requirements are Intel Xeon (R) CPUX5660@2.8GHz*12, NVIDIA Quadra4000 Graphics Card with 2048 MBRAM,32GB RAM,4.399.8GB Hard Disc The "Flight Data Visualization and Comprehensive Analysis Software" is an object oriented based software, which is integrated with existing "synthetic terrain visualization" software. The main objective of integrating the same is to carry out data analysis in on line, which is super imposed on the outside imagery generated using ADA software. 
The "synthetic terrain visualization" software is developed from open source graphical library, which is compiled using "GNU” compiler (GNU C++compiler, GNU C Compiler, GNU Fortran95 Compiler). It has a database of terrain imagery in "flight (FLT)" format, which has the details of the triangles and the polygons. The above software's are being developed using $\mathrm{C}++$ language under Windows platform. A make file is used to integrate and compile using GNU compiler.

\section{Experimental techniques}

\subsection{General}

FDVCAS is PC based 3d data analyzation software, which presents the aircraft parameters pictorially along with the outside view. Different views shall be selected namely, Pilot view, chase view to study the impact of the data on the system. It consists three main modules namely, Acquisition Module, Graphical Analyser module and Synthetic Terrain Visualization software module. FDVCAS displays STV by default and the graphical analyzer shall be displayed by pressing hot key F10. The display of Data display and warning display window shall also be controlled through GUI. The display of window shall be transparent through which the background shall be viewed. Acquisition Module acquires data from an offline data file in the form of ASCII in a Comma Separation Variable (CSV) format. The first row of the data file consists of the name of the data variable and followed rows represent its data. Each record in the data file terminates with the new line character. The selected data from the Acquisition Module passes the data to both Graphical Analyser Module and Synthetic Terrain Visualization Software Module. These modules help the user to view the data both in graphical format and to reconstruct the flight path with the outside window imagery.

Graphical analyser provides a menu bar to load the data file and helps to select different graphical user interfaces, namely graph display and data display, and also controls the slider window display. The graph view consists of different view types namely, full view, frame view and sine view. Plotting of the parameters shall be with respect to time and more than one parameters shall be plotted and the same in different colour. The selection and the deselection of the view shall be controlled through GUI.

\subsubsection{Full graph view}

Full graph view presents the full sortie of data in a single frame. $\mathrm{X}$-axis represents the time and both Y1 and Y2 axis represents the selected parameters. The display of number of Yaxis is based on the selection in the configure window. The memory is provided to hold 250 values of both Y1 and Y2. If the graph length is 250, then number of pixels to plot the graph is 250 so the graph can be plotted in maximum of 250 pixels. In order to map the entire data within the window having maximum of 250 pixels, the total record count for the sortie is calculated and 250 samples are picked from the total records for display. One pixel is equal to total record count divided by maximum available pixel. One pixel cannot hold more than one value so, only the first incoming value will be used for plotting. Depends on the range and the sampling rate of the variables the minimum and maximum value is fixed on the scale.

\subsubsection{Frame graph view}

In Frame graph $\mathrm{X}$-axis represents the time and both $\mathrm{Y} 1$ and $\mathrm{Y} 2$ axis represents the selected parameters. The Memory area for both Y-axis is created to hold 20 sample data. Every 
sample values are calculated based on the sample selected through Configure graph window. It provides selected variable list data to the Frame graph view. In Frame graph view variables are plotted for every 20 samples. As the data received for first 20 samples the graph is plotted i.e. (0 to 19). The next incoming sample is shifted to the previous position i.e. 19th position and 19th position sample is shifted to 18th position and the first samples goes out similarly it gets updated for a complete flight sortie. We can change the range and sample rate of the variables. Depends on the range and the sampling rate of the variables the minimum and maximum value is fixed on the scale.

\subsubsection{Sine graph view}

In Sine graph $\mathrm{X}$-axis represents the time and both $\mathrm{Y} 1$ and $\mathrm{Y} 2$ axis represents the selected parameter. The Memory area for both $\mathrm{Y}$-axis is created to hold 20 sample data. Every sample values are calculated based on the sample selected through Configure graph window. It provides selected variable list data to the Sine graph view. In Sine graph view variables are plotted for every 20 samples after plotting the first 20 sample normally, we are pushing the first 10 samples out and placing the next 10 sample to the first position, their after the graph is updated for every 10 samples first until full flight sortie.

Data window is selected through view menu. Enabling and disabling of data window is done through view menu. On selection the data window is displayed on the right top of the screen in blue color. The main parameters, which are required to be monitored along with graph and $3 \mathrm{~d}$ outside imagery, displayed on the data window.

\subsection{Input design}

FDVCAS requires inputs to update the visual coordinates, which in turn refresh the outside window imagery, to display the parameters in engineering format and to display the data in the graph are received from the data file (offline.csv). The information, which is required to display the graph namely, name of the parameter, range, sampling rate (Table 1) are selected through 'configure' user interface.

\subsubsection{Data file: offline.CSV}

The Input parameters in the data files are at different sampling rate namely $16 \mathrm{~Hz}, 4 \mathrm{~Hz}$ and $1 \mathrm{~Hz}$. The outside window imagery required updating at $60 \mathrm{~Hz}$ to get a smooth display. In order to refresh the data at the rate that of visual update, in between samples in the existing data are calculated in the data acquisition module dynamically.

It contains the parameters in the form of ASCII in a Comma Separation Variable (CSV) format. The above table shows the details of the data in the data file. The first row of the data file consists of the name of the data variable as listed in the structure namely; HR, MIN, SEC, MSEC, PHI, THETA, HEADING, AOA, PR_ALT, LATITUDE, LONGITUDE, UNDER CARRIAGE STATUS, LWOW, RWOW, NWOW and CAS, and the followed rows in the file represent its data. Each parameter is separated with comma and each record in the data file terminates with the new line character.

\subsubsection{GUI (Graphical User Interface)}

The main menu bar consists of Prepare GUI, using which the parameters for $\mathrm{x}$ and $\mathrm{Y}$ axis are configured. The record index also shall be dynamically modifiable through slider bar 
GUI. The slider bar GUI shall be selected through a hotkey 's'. On subsequent selection the slider get deselected.

\subsubsection{Configure GUI}

CONFIGURE GUI is used to prepare the parameter information to get the configuration of the parameter to display the same in the graph. In order to display the parameter information list namely parameter name and parameter range, the lists of the same is provided from the data file.

The variable list, range and the sampling rate are provided to the user, through which the user selects the required information. The selected input from the GUI is send to Graphical analyzer to display the parameter in the graph. The data from the GUI shall be dynamically selectable, accordingly the graph window also get updated. The configure GUI with $\mathrm{X}$ and $\mathrm{Y}$-axis configured to display the same in the graph window are given below.

\subsection{Output design}

FDVCAS provides the outputs through graph window and the data window. The screen shots of each window shall be outputted dynamically either to the file or to the printer. The printer is also configured in FDVCAS to take the output dynamically.

\subsubsection{Graph window}

The graph window is selected through main menu. It displays the name of the parameters in the selected color the value is displayed in the graph. It displays the parameter pictorially in frame view, full view and sine graph view. Two parameters shall be plotted on the graph and in different color. It is a transparent window through which the background imagery shall be seen. The format of the graph window is as given below.

\subsubsection{Data window}

The data window is selected through main menu. The parameters to be displayed in the data window are pre-configured and not modifiable dynamically. The important parameters, which are required to be viewed during the re-play, are displayed in the data window. It is a transparent window through which the background imagery shall be seen.

The important parameters namely time in the format of hour: minutes: seconds, Calibrated Air speed in kilometre per hour, Angle of attack in degrees, Altitude from terrain in kilometres, pitch in degrees and heading degrees are displayed along with graph and $3 \mathrm{~d}$ outside imagery, displayed on the data window.

Table 1. Variable List of FDVCAS.

\begin{tabular}{|c|c|c|c|c|c|}
\hline Sr. No & Parameter name & Parameter Range & Unit & Sample & Parameter Data Type \\
\hline 1 & Pitch & -180 to +180 & Deg & $16 \mathrm{~Hz}$ & Double \\
\hline 2 & Roll & -180 to +180 & Deg & $16 \mathrm{~Hz}$ & Double \\
\hline 3 & Theta & -180 to +180 & Deg & $16 \mathrm{~Hz}$ & Double \\
\hline 4 & Heading & -180 to +180 & Deg & $16 \mathrm{~Hz}$ & Double \\
\hline 5 & Latitude & 0 to 180 & Deg & $4 \mathrm{~Hz}$ & Double \\
\hline 6 & Longitude & 0 to 180 & Deg & $4 \mathrm{~Hz}$ & Double \\
\hline 7 & Altitude & 0 to 20 & $\mathrm{Km}$ & $16 \mathrm{~Hz}$ & Double \\
\hline 8 & Fuel Quantity & 0 to 10000 & $\mathrm{Ltr}$ & $16 \mathrm{~Hz}$ & Double \\
\hline 9 & Distance & 0 to 20000 & $\mathrm{Km}$ & $16 \mathrm{~Hz}$ & Double \\
\hline
\end{tabular}




\begin{tabular}{|c|c|c|c|c|c|}
\hline 10 & Thrust & 0 to 100 & $\%$ & $16 \mathrm{~Hz}$ & Double \\
\hline 11 & Mach No & 0 to 1.5 & -- & $16 \mathrm{~Hz}$ & Double \\
\hline 12 & Speed & 0 to 2000 & $\mathrm{Km} / \mathrm{H}$ & $16 \mathrm{~Hz}$ & Double \\
\hline 13 & Warning no & 0 to 200 & No & $1 \mathrm{~Hz}$ & Integer \\
\hline 14 & Warning category & 1 to 3 & No & $1 \mathrm{~Hz}$ & Integer \\
\hline 15 & X Acceleration & -1 to +1 & PiRad & $16 \mathrm{~Hz}$ & Double \\
\hline 16 & Y Acceleration & -1 to +1 & PiRad & $16 \mathrm{~Hz}$ & Double \\
\hline 17 & Z Acceleration & -1 to +1 & PiRad & $16 \mathrm{~Hz}$ & Double \\
\hline 18 & X Velocity & -1 to +1 & PiRad & $16 \mathrm{~Hz}$ & Double \\
\hline 19 & Y Velocity & -1 to +1 & PiRad & $16 \mathrm{~Hz}$ & Double \\
\hline 20 & Z Velocity & -1 to +1 & PiRad & $16 \mathrm{~Hz}$ & Double \\
\hline 21 & Pressure & 0 to 200 & Bar & $16 \mathrm{~Hz}$ & Double \\
\hline
\end{tabular}

\section{Results and discussion}

\subsection{Sample input file}

The following parameter values are recorded from FDR, table 2 shows the values of parameters in the data file. The first row of the data file consist of the name of the data variables as listed in the structure namely: HR, MIN, SEC, MSE, PHI, THETA, PSI, AOA, ALT, LAT ,etc Each parameter is separated with comma and each record in the data file terminates with the new line character.

Table 2. PARAMETER VALUES.

\begin{tabular}{|l|l|l|l|l|l|l|l|l|l|}
\hline HR & MIN & SEC & MSE & PHI & THETA & PSI & AOA & ALT & LAT \\
\hline 18 & 48 & 30 & 80 & -0.65 & -0.225 & 243.3 & 2.1 & 277.112 & 26.29949 \\
\hline 18 & 48 & 30 & 80 & -0.6 & -0.15 & 243.3 & 2.1 & 277.112 & 26.29948 \\
\hline 18 & 48 & 30 & 80 & -0.55 & -0.075 & 243.3 & 2.1 & 277.112 & 26.29947 \\
\hline 18 & 48 & 30 & 80 & -0.5 & 0 & 243.3 & 2.1 & 277.112 & 26.29946 \\
\hline 18 & 48 & 30 & 80 & -0.525 & 0.05 & 243.35 & 2.1 & 277.112 & 26.29946 \\
\hline 18 & 48 & 30 & 80 & -0.55 & 0.1 & 243.4 & 2.1 & 277.112 & 26.29945 \\
\hline 18 & 48 & 30 & 80 & -0.575 & 0.15 & 243.45 & 2.1 & 277.112 & 26.29944 \\
\hline 18 & 48 & 30 & 80 & -0.6 & 0.2 & 243.5 & 2.1 & 277.112 & 26.29944 \\
\hline 18 & 48 & 30 & 80 & -0.6 & 0.2 & 243.5 & 2.1 & 277.112 & 26.29943 \\
\hline 18 & 48 & 30 & 80 & -0.6 & 0.2 & 243.5 & 2.1 & 277.112 & 26.29942 \\
\hline 18 & 48 & 30 & 80 & -0.6 & 0.2 & 243.5 & 2.1 & 277.112 & 26.29941 \\
\hline 18 & 48 & 31 & 80 & -0.6 & 0.2 & 243.5 & 2.1 & 277.112 & 26.29941 \\
\hline 18 & 48 & 31 & 80 & -0.6 & 0.25 & 243.475 & 2.1 & 277.112 & 26.2994 \\
\hline 18 & 48 & 31 & 80 & -0.6 & 0.3 & 243.45 & 2.1 & 277.112 & 26.29939 \\
\hline 18 & 48 & 31 & 80 & -0.6 & 0.35 & 243.425 & 2.1 & 277.112 & 26.29938 \\
\hline 18 & 48 & 31 & 80 & -0.6 & 0.4 & 243.4 & 2.1 & 277.112 & 26.29938 \\
\hline 18 & 48 & 31 & 80 & -0.55 & 0.5 & 243.375 & 2.1 & 277.112 & 26.29937 \\
\hline 18 & 48 & 31 & 80 & -0.5 & 0.6 & 243.35 & 2.1 & 277.112 & 26.29936 \\
\hline 18 & 48 & 31 & 80 & -0.45 & 0.7 & 243.325 & 2.1 & 277.112 & 26.29935 \\
\hline 18 & 48 & 31 & 80 & -0.4 & 0.8 & 243.3 & 2.1 & 277.112 & 26.29935 \\
\hline 18 & 48 & 31 & 80 & -0.375 & 0.8 & 243.275 & 2.1 & 277.112 & 26.29934 \\
\hline 18 & 48 & 31 & 80 & -0.35 & 0.8 & 243.25 & 2.1 & 277.112 & 26.29933 \\
\hline 18 & 48 & 31 & 80 & -0.325 & 0.8 & 243.225 & 2.1 & 277.112 & 26.29932 \\
\hline 18 & 48 & 32 & 80 & -0.3 & 0.8 & 243.2 & 2.1 & 277.112 & 26.29932 \\
\hline 18 & 48 & 32 & 80 & -0.225 & 0.675 & 243.2 & 2.1 & 277.112 & 26.29931 \\
\hline 18 & 48 & 32 & 80 & -0.15 & 0.55 & 243.2 & 2.1 & 277.112 & 26.2993 \\
\hline 18 & 48 & 32 & 80 & -0.075 & 0.425 & 243.2 & 2.1 & 277.112 & 26.29929 \\
\hline
\end{tabular}




\subsection{Output snapshots}

FDVCAS (figure 4) has been developed to analyze and compare various parameters of flight sortie and the snapshots describing the features are shown below:

When Y2 is selected it will show the list of parameter, and the user can select a color so that the Y2 parameters will also be represented in the particular color (figure 1 and figure 2).

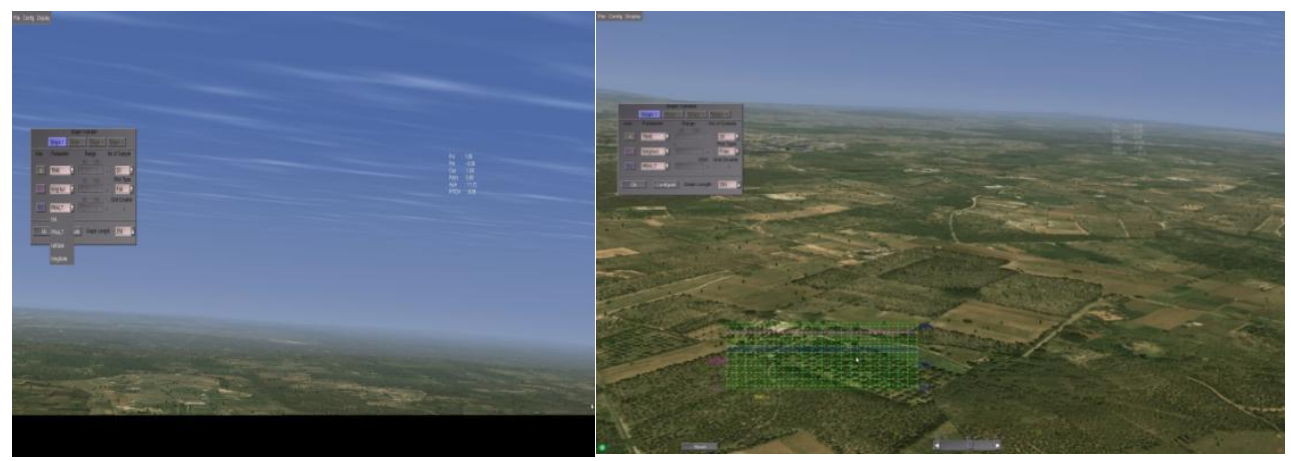

Fig. 1. The Y2 Parameter.

Fig. 2. Sine Graph is Viewed With Configure Window.


Fig. 3. Frame Graph View With Configure Window.

Fig. 4. Block diagram for FDVCAS.

Frame Graph Monitor updates the data every 20-milli seconds. X-axis represents the time at the bottom of the graph and it is shown in milliseconds and both Y1 and Y2 axis represents the parameter values. It provides selected variable list data to the Frame graph view. In Frame graph view (figure 3) variables are plotted for samples given the user. We can change the parameter, range and sample rate of the variables during the flight test. The existing parameters can be changed to the new parameter and again configured the graph will show the value of the new parameter.

\section{Conclusion and future scope}

FDVCAS is user-friendly data analysis software, successfully installed at data analysis division at ADA and working satisfactorily. Presently it is used to analyse the data for all the variants of LCA. The main advantage of this software is reduces the analysis time, improved efficiency, simple, user friendly and cost effective. The effort made to realize FDVCAS, yielded a very positive output, which became, one of very important product in the analysis software family of LCA. FDVCAS is fully operational and helping the ground grew to analyze the data effectively. Presently it has features to analyze the data graphically for a single configuration of an aircraft. Providing common analysis software would help 
the user to analyze the data's captured from different aircrafts, which made a sortie together. Also adding more intelligent would also help to analyze the software without any expert's presence

\section{References}

1. Guillermo Arango. Domain analysis methods. In Software Reusability, W. Schafer and R.Prieto-Diaz, editors, Ellis Horwood Publishers, 1993.

2. D. Batory, "Concepts for a Database System Synthesizer", Proc. ACM Principles of Database Systems, 1988.

3. D. Batory and S. O'Malley, "The Design and Implementation of Hierarchical Software Systems with Reusable Components", ACM Trans. Software Engineering and Methodology, October 1992.

4. D. Batory and J. Barnett, "DaTE: The Genesis DBMS Software Layout Editor", in Conceptual Modeling, Databases, and CASE: An Integrated View of Information Systems Development, P. Loucopoulos and R. Zicari, editors, Wiley, 1992.

5. D. Batory and S. Shafer, "A Domain Model for Avionics Software”, IBM Owego T.R. ADAGE-UT-93- 03, May 1993.

6. D. Batory, V. Singhal, M. Sirkin, and J. Thomas, "Scalable Software Libraries", Proceedings of ACM SIGSOFT 1993.

7. D. Batory, J. Thomas, and M. Sirkin, "Reengineering a Complex Application Using a Scalable Data Structure Compiler", Proceedings of ACM SIGSOFT 1994.

8. D. Batory and B. Geraci, "Validating Component Compositions in Software System Generators", Dept. Computer Sciences, University of Texas at Austin, TR-95-03.

9. K.H. Britton, R.A. Parker, and D.L. Parnas, "A Procedure for Designing Abstract Interfaces for Device Interface Modules”, Proceedings of ICSE 1981.

10. Lou Coglianese, et al., "An Avionics DomainSpecific Software Architecture," ARPA PI Conference, 1992. Also in CrossTalk, October 1992, and IBM Owego T.R. ADAGE-IBM-92-07, April 1992.

11. L. Coglianese and R. Szymanski, "DSSAADAGE: An Environment for Architecturebased Avionics Development", Proceedings of AGARD 1993. Also, IBM Owego T.R. ADAGE-IBM-93-04, May 1993.

12. D. Garlan, R. Allen, and J. Ockerbloom, "Architectural Mismatch or Why it's Hard to Build Systems out of Existing Parts", International Conference on Software Engineering, Seattle, Washington, 1995.

13. J. Goguen, "Reusing and Interconnecting Software Components", IEEE Computer, February 1986.

14. B. Curtis, H. Krasner, and N. Iscoe, "A Field Study of the Software Design Process for Large Systems", Communications of the ACM, November 1988. .

15. J V Muruga lal Jeyan and Akhila Rupesh, "Design, fabrication \& Systematic approach for Predicting aircraft fuel quantity at various maneuvering", International Journal Of advanced scientific and technical research, Vol.No. 4, Issue 4, PP: 325-238, July 30, 2014 , ISSN 2249-9954. 